\title{
Physiologically based pharmacokinetic and pharmacodynamic modeling of an antagonist (SM-406/AT-406) of multiple inhibitor of apoptosis proteins (IAPs) in a mouse xenograft model of human breast cancer
}

\author{
Tao Zhang ${ }^{\mathrm{a}, \dagger}$, Yanyan Li ${ }^{\mathrm{a}, \ddagger}$, Peng Zou ${ }^{\mathrm{a}}$, Jing-yu Yu ${ }^{\mathrm{a}}$, Donna McEachern ${ }^{\mathrm{b}}$, Shaomeng Wang ${ }^{\mathrm{b}}$, and Duxin Sun ${ }^{\mathrm{a}, *}$ \\ a Department of Pharmaceutical Sciences, University of Michigan, Ann Arbor, MI, 48109, USA \\ ${ }^{\mathrm{b}}$ Comprehensive Cancer Center and Departments of Internal Medicine, Pharmacology, and Medicinal Chemistry, University of Michigan, Ann \\ Arbor, MI, 48109, USA
}

\begin{abstract}
The inhibitors of apoptosis proteins (IAPs) are a class of key apoptosis regulators overexpressed or dysregulated in cancer. SM-406/AT-406 is a potent and selective small molecule mimetic of Smac that antagonizes the inhibitor of apoptosis proteins (IAPs). A physiologically based pharmacokinetic and pharmacodynamic (PBPK-PD) model was developed to predict the tissue concentration-time profiles of SM-406, the related onco-protein levels in tumor, and the tumor growth inhibition in a mouse model bearing human breast cancer xenograft. In the whole body physiologically based pharmacokinetic (PBPK) model for pharmacokinetics characterization, a well stirred (perfusion rate-limited) model was used to describe SM-406 pharmacokinetics in the lung, heart, kidney, intestine, liver and spleen, and a diffusion rate-limited (permeability limited) model was used for tumor. Pharmacodynamic (PD) models were developed to correlate the SM-406 concentration in tumor to the cIAP1 degradation, pro-caspase 8 decrease, CL-PARP accumulation and tumor growth inhibition. The PBPK-PD model well described the experimental pharmacokinetic data, the pharmacodynamic biomarker responses and tumor growth. This model may be helpful to predict tumor and plasma SM-406 concentrations in the clinic. Copyright (c) 2013 John Wiley \& Sons, Ltd.
\end{abstract}

Key words: pharmacodynamic modeling; SM-406/AT-406; multiple inhibitor of apoptosis proteins (IAPs); mouse xenograft model; human breast cancer

\section{Introduction}

Apoptosis (programmed cell death) is a critical cellular process to maintain normal development and homeostasis of multicellular organisms. Dysregulation of apoptosis has been implicated

*Correspondence to: Department of Pharmaceutical Sciences, University of Michigan, Ann Arbor, MI 48109, USA.

E-mail: duxins@umich.edu

${ }^{\dagger}$ Current address: DMPK, Novartis Institute for Biomedical Research, Novartis Pharmaceuticals Corporation, East Hanover, NJ, 07936, USA

${ }^{\ddagger}$ Current address: Department of Health and Nutrition Sciences, Montclair State University, Montclair, NJ, 07043, USA in many human diseases including cancer [1-3]. One of the key features that distinguish cancer cells from normal cells is apoptosis avoidance and resistance, which has been a major hurdle to current therapies [4]. Therefore, targeting crucial apoptosis regulators to overcome the evasion of cancer cell death has emerged as a promising therapeutic strategy.

A class of key apoptosis regulators has been identified, which are named as the inhibitors of apoptosis proteins (IAPs), including X-linked IAP (XIAP), cellular IAP1 and IAP2 (cIAP1 and cIAP2) [5-7]. $\mathrm{XIAP}$ is a central regulator of both death receptor mediated and mitochondria mediated apoptosis 
pathways, inhibiting apoptosis by directly binding to caspase-3, caspase-7 and caspase-9 [7]. The cIAP1 and CIAP2 play a crucial role in regulating tumor necrosis factor receptor - associated factor (TRAF) mediated apoptosis and suppressing caspase- 8 activation [5-7]. Second mitochondria derived activator of caspases (Smac) protein, also known as DIABLO (direct inhibitor of apoptosis-binding protein with low $\mathrm{pI}$ ), has been identified as an endogenous antagonist of IAP proteins [8,9]. It has been proposed that SMAC interacts with XIAP and cIAP1/ 2 proteins via its Ala-Val-Pro-Ile (AVPI) tetrapeptide binding motif to promote the activation of caspases and to induce apoptosis [10-13].

SM-406/AT-406 is a small molecule mimetic of Smac currently being evaluated in clinical phase I studies as an anticancer agent [14]. It was designed based upon the crystal structure of Smac and confirmed to be a selective and potent antagonist of the IAP proteins [14]. SM-406 bound to XIAP, cIAP1 and cIAP2 with $K_{\mathrm{i}}$ of $66.4,1.9$ and $5.1 \mathrm{nM}$ [14]. In a cell-free functional assay, SM-406 completely antagonized XIAP and restored the activity of caspase-9 at $1 \mu \mathrm{M}$ [14]. In human breast cancer cells, it rapidly and effectively induced cIAP1 degradation at $100 \mathrm{nM}$ [14]. SM-406 exhibited moderate bioavailability after oral administration in the mice, rat, dog and non-human primates [14].

In the drug discovery and development process, pharmacokinetic-pharmacodynamic (PKPD) modeling has been applied increasingly, particularly for selecting drug candidates with favorable PK-PD properties and optimizing study design. It is not only a valuable tool to understand the pharmacokinetic property and therapeutic response of an existing compound, but can also provide important information for evaluating new chemical entities. Generally the PK-PD relationship is established with preclinical data to obtain an insight of the drug action mechanism in vivo. The commonly used methodologies for PK prediction are allometric scaling and physiologically based pharmacokinetic (PBPK) modeling. PBPK models are mathematical models derived from the anatomical and physiological structure of the organism studied [15]. PBPK models are considered to be mechanistically based, because they reflect our knowledge of the mechanisms of the underlying pharmacokinetic process. Although the establishment of PBPK models can be tedious and requires extensive effort of both physiological and drug specific parameters input and mathematical analysis, it offers the major advantage of greater extrapolation power [15]. Pharmacodynamic studies can provide information on the mechanism or dose-response relationship of a drug, and the response can be classified as biomarkers, surrogate end points or clinical end points [16]. By building a PK-PD model, the kinetic information can be related to the observed effect and provide a better understanding of the drug exposure and sequence of the events resulting in the drug effect [16].

The objective of the present study was to investigate the PK-PD relationship of SM-406 in mice bearing human breast cancer xenografts. A PBPK model was developed to describe the concentration-time profiles of SM-406 in the tissues, and a PD model was established to characterize the biomarker response in tumor and tumor growth kinetics. Finally, an integrated PBPK-PD model was generated to give a full pharmacokinetic and efficacy profile of SM-406 in vivo.

\section{Materials and Methods}

\section{Chemicals}

SM-406 and an internal standard (IS) SM-408 for LC-MS/MS were synthesized as described previously [14]. Acetonitrile and methanol of HPLCgrade were purchased from Fisher Scientific (Pittsburgh, PA, USA). Distilled water was produced in house by a Milli-Q water purification system (Millipore Corp., Bedford, MA). Ammonium acetate (HPLC-grade) and formic acid (spectroscopic grade) were purchased from Sigma/Aldrich (MO, USA).

\section{In vivo pharmacokinetics studies}

The mouse model bearing human MDA-MB-231 xenograft tumor was employed in this study. To develop xenograft tumors, $5 \times 10^{6}$ MDA-MB-231 cancer cells were mixed with matrigel and injected subcutaneously on the dorsal side of severe combined immunodeficient SCID mice (Charles River Laboratories, Wilmington, MA). SM-406 was dissolved in PBS and the $\mathrm{pH}$ was adjusted with $\mathrm{NaOH}$ as needed. The $\mathrm{pH}$ of the drug solutions was checked before administration to ensure it was between pH3.0 and 9.0 for oral administration 
and between $\mathrm{pH} 4.5$ and 9.0 for intravenous administration. Mice bearing MDA-MB-231 xenograft tumors were administered with control vehicle or a single intravenous dose of SM-406 at $10 \mathrm{mg} / \mathrm{kg}$, single oral doses of SM-406 at 30 and $100 \mathrm{mg} / \mathrm{kg}$ delivered by oral gavage. There were three mice per time point. Blood samples were collected into heparinized tubes from each mouse by terminal cardiac puncture at $0.25,0.5,1,2,4,6,8,24 \mathrm{~h}$ postdose. The plasma fraction was immediately separated by centrifugation at $4{ }^{\circ} \mathrm{C}$ and stored at $-80^{\circ} \mathrm{C}$ until analysis. For the $100 \mathrm{mg} / \mathrm{kg}$ group, liver, lung, heart, kidney, tumor, spleen and intestine were also immediately dissected, weighed, snap-frozen and ground with a mortar and pestle in liquid nitrogen, and then stored at $-80^{\circ} \mathrm{C}$ until analysis. An additional two groups of mice (3 or 4 per group) were dosed and placed in metabolic cages (Harvard Apparatus, South Natick, MA) for separate collection of urine and feces. All animal experiments were performed under the guidelines of the University of Michigan Committee for Use and Care of Animals.

The quantitative determination of the plasma, tissue, urine and feces concentration of SM-406 was done with LC-MS/MS using an Agilent 1200 HPLC system coupled to an API 3200 mass spectrometer (Applied Biosystems, MDS Sciex Toronto, Canada) equipped with an API electrospray ionization (ESI) source as described previously [14]. The mass spectrometer was operated at ESI positive ion mode and the detection of the ions was performed in the multiple reaction monitoring (MRM) mode, monitoring the transition of $\mathrm{m} / z 562.3$ precursor ion $[\mathrm{M}+\mathrm{H}]+$ to the $m / z 167.1$ product ion for SM406 and $m / z 610.2$ precursor ion $[\mathrm{M}+\mathrm{H}]+$ to the $m / z 167.1$ product ion for internal standard (SM408). Mobile phase A and B were water and methanol, and both contained $5 \mathrm{mM}$ ammonium acetate and $0.1 \%$ formic acid $(\mathrm{v} / \mathrm{v})$. The flow gradient was initially $90: 10 \mathrm{v} / \mathrm{v}$ of $A: B$ for 2 min, linearly ramped to $0: 100$ over $4 \mathrm{~min}$, held at 0:100 for $2 \mathrm{~min}$, and then returned to $90: 10$ over $0.1 \mathrm{~min}$. This condition was held for a further $5 \mathrm{~min}$ prior to the injection of another sample. The ion spray voltage was set at $5500 \mathrm{~V}$. The ionization temperature was set as $700^{\circ} \mathrm{C}$. The instrument parameters, curtain gas, gas 1 and gas 2 (auxillary gas), were set at 20, 60 and 60 , respectively. The compounds parameters, declustering potential (DP), collision energy
(CE), entrance potential (EP), collision entrance energy (CEP) and collision exit potential (CXP) were $61,45,5,26,4 \mathrm{~V}$ and $61,45,9,27.3,4 \mathrm{~V}$ for SM-406 and IS (SM-408), respectively. Data acquisition and quantitation were performed using analyst software version 1.4.2 (Applied Biosystems, MDS Sciex Toronto, Canada).

Plasma samples were prepared by mixing plasma with acetonitrile containing IS. The solution was vortexed for $1 \mathrm{~min}$ at high speed and centrifuged at $13000 \mathrm{rpm}$ for $10 \mathrm{~min}$ to precipitate protein. The clear supernatant was transferred to vial inserts for LC-MS/MS analysis. Calibration standard solutions were prepared with blank plasma. The lower limit of quantification was $1 \mathrm{ng} / \mathrm{ml}$. Intraday variability was assessed through analysis of QCs in triplicate and the inter-day variability was determined by analysing QCs on three different days, and the $C V \%$ was less than $5 \%$. To prepare tissue samples, about $60-80 \mathrm{mg}$ of ground tissue powder was weighed and mixed with PBS to obtain final concentrations (w/v) of $200 \mathrm{mg} / \mathrm{ml}$. Samples were homogenized using a tissue homogenizer (Tissuemiser Homogenizer, Fisher Scientific) for $20 \mathrm{~s}$. Then the homogenate was treated similarly as for plasma for LC-MS/MS analysis. Calibration standard solutions were prepared with blank tissue homogenate. The lower limit of quantification for tissue samples ranged from 2.5 to $12.5 \mathrm{ng} / \mathrm{g}$. Urine samples were processed similarly to plasma, and feces were homogenized first before subjecting to a similar procedure.

\section{In vivo pharmacodynamic studies}

Mice bearing MDA-MB-231 xenografts tumors were orally administered with a single dose of $100 \mathrm{mg} / \mathrm{kg}$ SM-406, and tumor tissues were harvested at $2 \mathrm{~h}, 6 \mathrm{~h}, 16 \mathrm{~h}$ and $24 \mathrm{~h}$. The tumor tissues for mice administered with control vehicle were collected at $24 \mathrm{~h}$. Tumor tissues were lysed using radioimmunoprecipitation assay (RIPA) lysis buffer (PBS containing 1\% NP-40, 0.5\% sodium deoxycholate and $0.1 \%$ sodium dodecyl sulfate [SDS]) supplemented with $1 \mu \mathrm{M}$ phenylmethylsulfonyl fluoride and one protease inhibitor cocktail tablet per $10 \mathrm{ml}$ on ice for $20 \mathrm{~min}$, and lysates were then cleared by centrifugation prior to protein concentration determination using BCA assay kit (Pierce, Rockford, IL) 
according to the manufacturer's instructions. Proteins were electrophoresed onto 18\% SDSPAGE gels (Invitrogen, Grand Island, NY) and transferred to PVDF membranes. Following blocking in 5\% milk, the membranes were incubated with a specific primary antibody, washed and incubated with horseradish peroxidase linked secondary antibody (Amersham, Piscataway, NJ). The signals were visualized with the chemiluminescent HRP antibody detection reagent (Denville Scientific, Metuchen, NJ). When indicated, the blots were stripped and reprobed with a different antibody. The following primary antibodies were used in the study: anti-cIAP (from R\&D), anti-procaspase-8 (Stressgen Biotechnologies, Victoria, Canada) and anti-PARP (Cell Signaling Technology, Beverly, MA). Quantification of western blotting bands was performed by densitometry analysis with Image J software (NIH, Bethesda, MD).

To determine the antitumor activity of SM-406, SCID mice bearing MDA-MD-231 xenograft tumors were orally administered vehicle, $30 \mathrm{mg} / \mathrm{kg}$ and $100 \mathrm{mg} / \mathrm{kg}$ of SM-406 daily (5 days per week) for 2 weeks. The tumor length and width were measured using a digital caliper 2-3 times a week and tumor volumes were calculated using the formula, volume $\left(\mathrm{mm}^{3}\right)=L \times W^{2} / 2$ (length $L, \mathrm{~mm}$; width $W$, $\mathrm{mm}$ ) as described previously [17].

\section{Blood distribution and protein binding}

Plasma protein binding was measured as described previously [18]. Plasma was spiked with SM-406 to yield a final concentration of $1 \mu \mathrm{g} / \mathrm{ml}$ and $5 \mu \mathrm{g} / \mathrm{ml}$. After incubation at $37^{\circ} \mathrm{C}$ for $1 \mathrm{~h}$, an aliquot was transferred to a $10 \mathrm{kD}$ cut-off ultrafiltration device (Millipore Corporation, Billerica, MA, USA), and was centrifuged at $1500 \times g$ for $1 \mathrm{~h}$ at $37^{\circ} \mathrm{C}$. The samples were analysed by LCMS/MS as described above. The concentrations of SM-406 in the filtrate were determined by a standard curve constructed with known concentrations of SM-406 in protein-free plasma (macromolecules had been removed from the plasma by $10 \mathrm{kD}$ cut-off ultrafiltration). The unbound fraction was estimated from the ratio of SM-406 concentration in the filtrate to that in the original plasma samples. Compound dissolved in protein-free plasma was studied in parallel to show that SM-406 binds minimally to the ultrafiltration device. The calculated unbound fraction in plasma $\left(f u_{\mathrm{p}}\right)$ at $1 \mu \mathrm{g} / \mathrm{ml}$ is 0.185 and at $5 \mu \mathrm{g} / \mathrm{ml}$ is 0.195 , and the average of 0.19 was used in further studies.

To determine blood to plasma partitioning $\left(K_{\mathrm{b} / \mathrm{p}}\right), \mathrm{SM}-406$ (1 and $\left.5 \mu \mathrm{g} / \mathrm{ml}\right)$ was added to mice blood $(2 \mathrm{ml})$ and incubated at $37^{\circ} \mathrm{C}$ for $1 \mathrm{~h}$ with very gentle shaking. After incubation, aliquots of blood $(50 \mu \mathrm{l})$ were removed, and the remaining blood was centrifuged at $1000 \times g$ for $10 \mathrm{~min}$ to separate plasma. The concentrations of SM-406 in whole blood and plasma were determined using LC-MS/MS and separate calibration curves were constructed with blank blood and plasma spiked with known concentrations of SM-406. The blood to plasma ratio $\left(K_{\mathrm{b} / \mathrm{p}}\right)$ is determined by the drug concentration in blood compared with plasma. The blood to plasma ratio $\left(K_{\mathrm{b} / \mathrm{p}}\right)$ at $1 \mu \mathrm{g} / \mathrm{ml}$ is 1.02 and at $5 \mu \mathrm{g} / \mathrm{ml}$ is 1.06 , so an average of 1.04 was used. The concentration of SM-406 in venous blood was calculated from: $C_{\text {ven }}(\mathrm{t})=C_{\mathrm{p}}(\mathrm{t})^{*} K_{\mathrm{b} / \mathrm{p}}$, where $C_{\mathrm{p}}$ is the plasma concentration.

\section{Physiologically based pharmacokinetic model}

Local models were built with blood and tissue concentration-time data after the oral administration of $100 \mathrm{mg} / \mathrm{kg}$ SM-406. The plasma drug concentration-time curve declines in biexponential manner, so in local models, a commonly used two-compartment model with extravascular administration was employed to describe the plasma concentration-time data. The plasma model used first order absorption and elimination. Both perfusion rate-limited and diffusion rate-limited models were evaluated for each tissue and the selection of final models was based on the quality of fits and the Akaike information criteria.

The mass-balance differential equations for well stirred organs (lung, heart, spleen, intestine, kidney, muscle) were listed below:

$$
V_{\text {Tissue }} \bullet \frac{d C_{\text {Tissue }}}{d t}=Q_{\text {Tissue }} \bullet\left(C_{\text {art }}-\frac{C_{\text {Tissue }}}{R_{\text {Tissue }}}\right)
$$

The mass-balance differential equations for diffusion-limited organs (tumor, rest of the body) were:

Biopharm. Drug Dispos. 34: 348-359 (2013) DOI: $10.1002 /$ bdd 


$$
\begin{gathered}
V_{\text {Tissue_v }} \bullet \frac{d C_{\text {Tissue_v }}}{d t}=Q_{\text {Tissue }} \bullet\left(C_{\text {art }}-C_{\text {Tissue_v }}\right)-P S_{\text {Tissue }} \\
\bullet f u b \bullet\left(C_{\text {Tissue_v }}-\frac{C_{\text {Tissue_ev }}}{R_{\text {Tissue }}}\right) \\
V_{\text {Tissue_ev }} \bullet \frac{d C_{\text {Tissue_ev }}}{d t}=P S_{\text {Tissue }} \bullet f u b \bullet\left(C_{\text {Tissue_v }}-\frac{C_{\text {Tissue_ev }}}{R_{\text {Tissue }}}\right)
\end{gathered}
$$

Where $Q_{\text {Tissue }}$ represents organ blood flow rate, $V_{\text {Tissue }}$ represents organ volume, $V_{\text {Tissue_v }}$ represents the volume of vascular space, $V_{\text {Tissue_ev }}$ represents the volume of extravascular space, $C_{\text {art }}$ represents the input from arterial blood, $f_{\mathrm{ub}}$ represents the free fraction in blood. For lung organ, the input is the output from blood: $Q_{\text {Lung }}{ }^{*} C_{\text {ven }} P S_{\text {Tissue }}$ represents permeability surface area product, and $R_{\text {Tissue }}$ represents the partitioning coefficient.

The mass-balance differential equations for eliminating organs liver and kidney were listed below:

$$
\begin{aligned}
V_{\mathrm{li}} \bullet \frac{d C_{\mathrm{li}}}{d t}= & Q_{\mathrm{sp}} \bullet \frac{C_{\mathrm{sp}}}{R_{\mathrm{sp}}}+Q_{\mathrm{in}} \cdot \frac{C_{\mathrm{in}}}{R_{\mathrm{in}}}+\left(Q_{\mathrm{hp}}-Q_{\mathrm{sp}}-Q_{\mathrm{in}}\right) \\
& \bullet C_{\mathrm{art}}-Q_{\mathrm{hp}} \bullet \frac{C_{\mathrm{li}}}{R_{\mathrm{li}}}-f_{\mathrm{ub}} \bullet C L_{\mathrm{int}} \frac{C_{\mathrm{li}}}{R_{\mathrm{li}}} \\
V_{\mathrm{ki}} \bullet \frac{d C_{\mathrm{ki}}}{d t}= & Q_{\mathrm{ki}} \bullet C_{\mathrm{art}}-Q_{\mathrm{ki}} \bullet \frac{C_{\mathrm{ki}}}{R_{\mathrm{ki}}}-C L_{\mathrm{r}} \bullet C_{\mathrm{ki}}
\end{aligned}
$$

Where $Q_{\mathrm{sp}}, Q_{\mathrm{in}} Q_{\mathrm{hp}}, Q_{\mathrm{ki}}$ represent the spleen, intestine, hepatic and kidney blood flow rate, $V_{\text {li }}$ and $V_{\mathrm{ki}}$ represent liver and kidney volume, $C_{\mathrm{sp}}$, $C_{\mathrm{in}}, C_{\mathrm{li}}, C_{\mathrm{ki}}$ and $C_{\text {art }}$ represent drug concentrations in each organ, $C L_{\text {int_li }}$ represents the intrinsic clearance with respect to free drug concentration in the liver, and $C L_{\mathrm{r}}$ represents renal clearance.

The physiologic parameters were either obtained from the literature or determined by experiment and listed in Table 1 [19-21]. The initial estimates of the partitioning coefficient were determined by the ratio of the area under concentration curves (AUC) between tissue and blood. The AUC values were calculated using noncompartmental analysis with WinNonlin software (Pharsight Corporation, Cary, NC, USA). In nonsampled muscle tissue the value was estimated with the PBPKPlus ${ }^{\mathrm{TM}}$ module of Gastroplus software, which incorporated the physiochemical property such as $\log \mathrm{P}, \mathrm{pKa}$ and
Table 1. Physiological parameters used in the whole body PBPK model for tumor-bearing mice

\begin{tabular}{lccc}
\hline & $\begin{array}{c}\text { Total volume } \\
(\mathrm{ml})\end{array}$ & $\begin{array}{c}\text { Vascular } \\
\text { volume } \\
\text { (as \% of } \\
\text { organ volume) }\end{array}$ & $\begin{array}{c}\text { Organ blood } \\
\text { flow } \\
\text { (\% cardiac } \\
\text { output) }\end{array}$ \\
\hline Whole body & $19.74^{\mathrm{a}}$ & - & - \\
Blood & $1.003^{\mathrm{b}}$ & - & 100 \\
Lung & $0.155^{\mathrm{a}}$ & - & 100 \\
Liver & $1.147^{\mathrm{a}}$ & - & $16.1^{\mathrm{b}}$ \\
Spleen & $0.034^{\mathrm{a}}$ & - & $0.42^{\mathrm{d}}$ \\
Intestine & $1.113^{\mathrm{a}}$ & - & $11.99^{\mathrm{d}}$ \\
Heart & $0.114^{\mathrm{a}}$ & - & $6.6^{\mathrm{b}}$ \\
Kidney & $0.324^{\mathrm{a}}$ & - & $9.1^{\mathrm{b}}$ \\
Tumor & $0.475^{\mathrm{a}}$ & $10^{\mathrm{c}}$ & $1.73^{\mathrm{c}}$ \\
Muscle & $7.860^{\mathrm{b}}$ & - & $15.9^{\mathrm{b}}$ \\
Rest of body & $8.245^{\mathrm{a}}$ & $10^{\mathrm{c}}$ & 50.57 \\
\hline
\end{tabular}

${ }^{a}$ Determined by averaging the experimental data for each organ and assuming a density of $1 \mathrm{~g} / \mathrm{ml}$ except for bone, $1.5 \mathrm{~g} / \mathrm{ml}$; average body weight $(\mathrm{BW})=20.47 \mathrm{~g}$.

bValues obtained from Brown et al. [21].

${ }^{c}$ Values obtained from Baxter et al. [20].

${ }^{\mathrm{d}}$ Values obtained from Wang et al. [19], cardiac output $=51.36 \mathrm{ml} / \mathrm{min} /$ $100 \mathrm{~g} \mathrm{BW}$.

plasma protein binding of the compound (Simulations Plus Inc., Lancaster, USA). The parameters obtained from individual models were used as initial estimates for the whole body model. The whole body PBPK model depicts the body as being composed of nine tissue compartments and one blood compartment (Figure 1). Oral administration was treated as a bolus into a dummy depot compartment from which a fraction of the dose $(F \%)$ could enter the blood with the first-order rate constant. All equations were solved simultaneously with the maximum likelihood estimator in the ADAPT 5 software [22]. The variance model was defined as: $V A R_{\mathrm{i}}=\left(\sigma_{\text {inter }}+\sigma_{\text {slope }} \cdot Y_{i}\left(t_{j}\right)\right)^{2}$, where $V A R_{\mathrm{i}}$ is the variance of the $i$ th data point, $\sigma_{\text {inter }}$ $\sigma_{\text {slope }}$ are the variance model parameters. The estimated values for $\sigma_{\text {inter }}$ and $\sigma_{\text {slope }}$ are 0.10 and 0.53 . The bioavailability was obtained by comparing the plasma AUC of oral dose $(100 \mathrm{mg} / \mathrm{kg})$ with the intravenous dose $(10 \mathrm{mg} / \mathrm{kg})$.

\section{Pharmacodynamic model}

To characterize the relationship between the SM406 concentration and efficacy, an integrated PBPK-PD model (Figure 2) was established using the PBPK estimated tumor SM-406 concentration as an input to modulate onco-protein levels and 


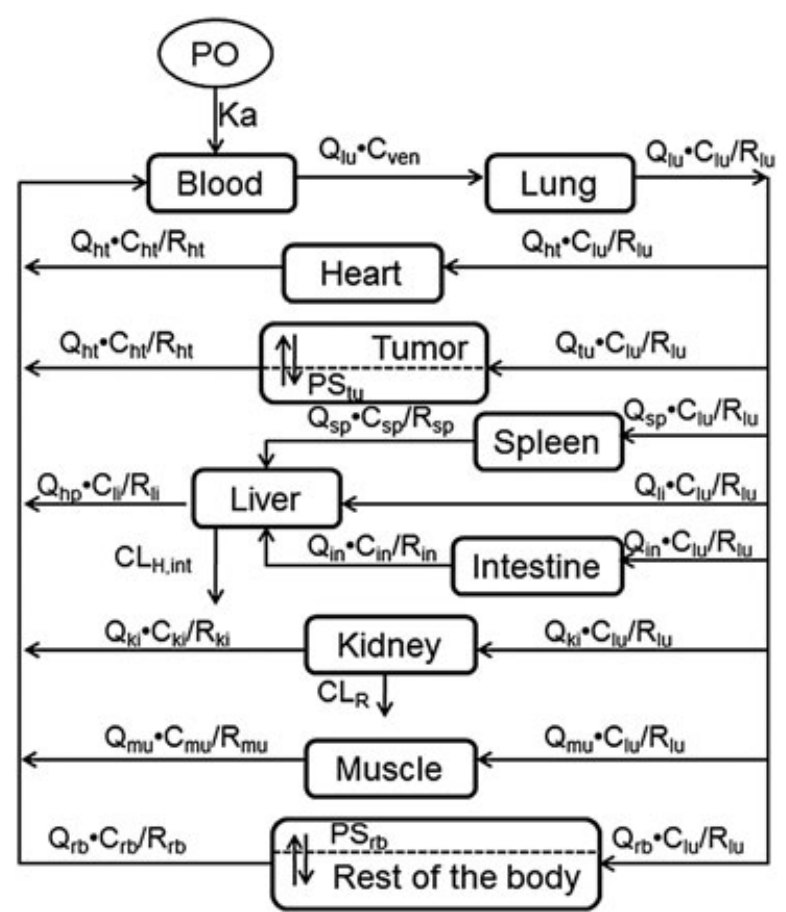

Figure 1. Whole body PBPK model for SM-406 following an oral dose. Subscripts are: ven, venous blood; lu, lung; ht, heart; tu, tumor; li, liver; sp, spleen; in, intestine; ki, kidney; mu, muscle; rb, rest of the body

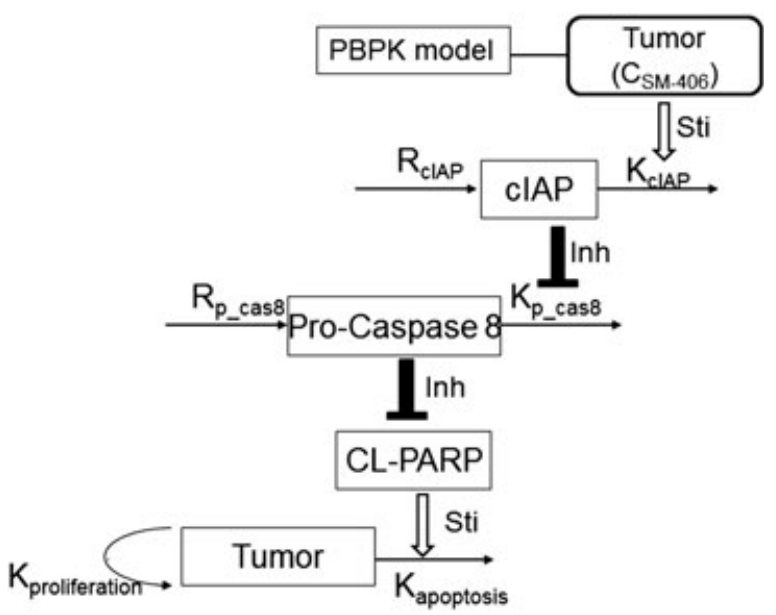

Figure 2. Schematic illustration of the caspase activation and apoptosis pathway model linking tumor SM-406 concentration $\left(C_{\mathrm{SM}-406}\right)$ to onco-protein levels and tumor growth inhibition. cIAP, cellular inhibitors of apoptosis proteins; CL-PARP, cleaved PARP; Sti, stimulation; Inh, inhibition tumor growth inhibition. The PD model was built based on the molecular mechanism of caspase activation and apoptosis pathways with some modifications [23,24]. The inhibition/stimulation of onco-protein degradation or tumor apoptosis was described by the following equations:

$$
\begin{aligned}
\frac{d c I A P I}{d t} & =R_{\text {syn_cIAP1 }} \\
& -K_{\text {deg_cIAP1 }} \bullet C I A P I \bullet\left(1+\frac{E_{\text {max_CIAP1 }} \bullet C_{\text {tumor }}}{E C_{50 \_c I A P 1}+C_{\text {tumor }}}\right)
\end{aligned}
$$

Where $R_{\text {syn_cIAP1 }}$ is the zero order formation of CIAP1, $K_{\text {deg_cIAP1 }}$ is the first order rate constant defining the degradation of CIAP1, $E_{\text {max_cIAP1 }}$ is the maximum stimulatory effect of SM- 406 on cIAP1 degradation, $E C_{50 \_c I A P 1}$ is the concentration producing 50\% stimulatory effect. The level of cIAP1 at baseline is set at 1 , so $R_{\text {syn_cIAP1 }}=K_{\text {deg_cIAP1 } 1}$.

$$
\begin{aligned}
\frac{d p C A S 8}{d t} & =R_{\text {syn_pCAS8 }} \\
& -K_{\text {deg_pCAS8 }} \bullet p C A S 8 \bullet\left(1-K_{\text {pCAS8 } 8} \bullet(\text { cIAPI- } 1)\right)
\end{aligned}
$$

Where $R_{\text {syn_pCAS8 }}$ is the zero order formation of pCAS8, $K_{\text {deg_pCAS8 }}$ is the first order rate constant defining the degradation of pCAS8, $K_{\mathrm{pCAS}}$ is the constant describing the effect of cIAP1 on pCAS8 degradation. The baseline level of pCAS8 is set at 1 , so $R_{\text {syn_pCAS8 }}=K_{\text {deg_pCAS8 }}$.

$$
C L \_P A R P=\text { Inter }_{\text {CL_PARP }}-\text { Slope }_{\text {CL_PARP }} \bullet p C A S 8
$$

Where the CL_PARP and pCAS8 levels are described by a linear relationship based on the data. The baseline level of CL_PARP is set at 1, so Inter $_{\text {CL_PARP }}=$ Slope $_{\text {CL_PARP }}+1$.

The tumor growth model was built on a simple exponential growth modified with a logistic function containing a maximum tumor volume $\left(T V_{\max }\right)[25]$.

$$
\begin{aligned}
\frac{d T V}{d t} & =R_{\text {tu_grow }} \bullet T V \bullet\left(1-\frac{T V}{T V_{\max }}\right) \\
& -T V \bullet C L \_P A R P \bullet K_{\text {tu_apop }}
\end{aligned}
$$

Where $T V$ is tumor volume, $R_{\text {tu_grow }}$ and $K_{\text {tu_grow }}$ correspond to tumor growth and apoptosis rate, $T V_{\max }$ is the maximum tumor volume. When tumor is very small, the ratio of $T V / T V_{\max }$ is close 
to zero and tumor grows with approximate firstorder rate; when tumor reaches maximum size, the ratio of $T V / T V_{\max }$ approximates 1 and there is no more net growth. The initial estimates for tumor growth parameters were generated with WinNonlin (Pharsight Corp., Mountain View, CA) software using tumor size data from control, $30 \mathrm{mg} / \mathrm{kg}$ and $100 \mathrm{mg} / \mathrm{kg}$ treatments simultaneously and the whole PBPK-PD model was built with the ADAPT 5 software [22].

\section{Results}

\section{PBPK model}

The plasma and tissue concentration-time profiles of observed data after $100 \mathrm{mg} / \mathrm{kg}$ dose of SM-406 were shown in Figure 3 (solid circles). The whole body PBPK model for SM-406 in MDA-MD-231 xenograft tumor bearing SCID mice is depicted in Figure 1. The physiological parameters for the whole body PBPK model were either obtained from the literature or determined by experiment and are listed in Table 1 . The blood to plasma ratio $\left(K_{\mathrm{b} / \mathrm{p}}\right)$ was 1.04 and the unbound fraction in plasma $\left(f_{\text {up }}\right)$ was 0.19 . All equations were solved simultaneously with the maximum likelihood estimator in the ADAPT 5 software [22] and the fitted concentration-time profiles are shown in Figure 3 (solid lines). It appears from the graphs that the model captured the plasma concentration-time profiles quite well. After oral administration, the estimated first-order absorption rate constant was $0.974 \mathrm{~h}^{-1}$.

Concentration-time profiles for lung, heart, kidney, intestine, liver and spleen captured by the PBPK model are also depicted in Figure 3 (solid lines). Concentration-time curves of these organs declined approximately in parallel with that of blood, so the well stirred model was used. The model depicted the observed data relatively well for most organs. The tissue-to-blood partition coefficient $\left(R_{\mathrm{T}}\right)$ calculated by noncompartmental analysis and those estimated from the PBPK model are reported in Table 2. Model-defined $R_{\mathrm{T}}$ values were obtained with good precision with $\mathrm{CV} \%$ less than $20 \%$. The deviation between the noncompartmental and PBPK-defined $R_{\mathrm{T}}$ values never exceeded 1.5-fold. The model obtained liver intrinsic clearance was $685 \mathrm{ml} / \mathrm{h}$, which corresponded to a systemic liver clearance of $40.8 \mathrm{ml} / \mathrm{h}$. The renal clearance of $7.6 \mathrm{ml} / \mathrm{h}$ was calculated based on the total amount of drug recovered

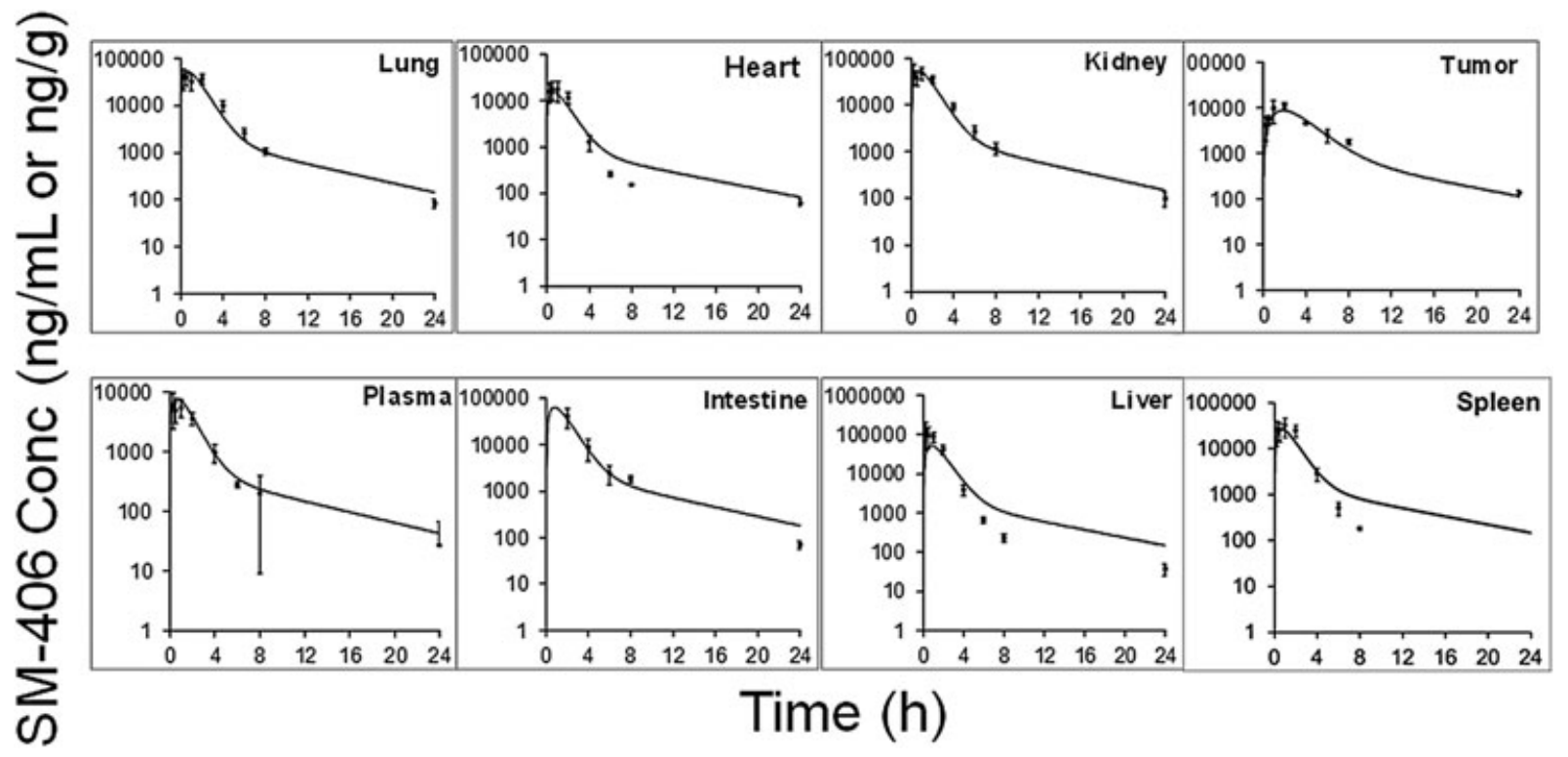

Figure 3. Plasma and tissue concentration-time profiles of SM-406 following an oral dose (100 mg/kg) in tumor-bearing mice. Solid circles represent observed concentrations (mean $\pm \mathrm{SD}, n=3$ ) and the lines are model-fitted profiles 
Table 2. Physiological pharmacokinetic parameters for SM-406 using the whole body PBPK model

\begin{tabular}{|c|c|c|c|}
\hline Parameter & Estimated value & $\mathrm{CV} \%$ & Partition coefficient $(R)$ by $A U C$ ratio $^{c}$ \\
\hline$f_{\text {uplasma }}$ & $0.19^{\mathrm{a}}$ & - & - \\
\hline F\% (bioavailability) & $42.7 \%{ }^{\mathrm{a}}$ & - & - \\
\hline$K_{\mathrm{a}}(1 / \mathrm{h})$ & 0.974 & 6.52 & - \\
\hline$R_{\text {lung }}$ & 6.0 & 12.4 & 7.73 \\
\hline$R_{\text {heart }}$ & 1.86 & 12.4 & 2.57 \\
\hline$R_{\text {tumor }}$ & 2.24 & 7.19 & 3.17 \\
\hline$R_{\text {kidney }}$ & 6.4 & 12.4 & 8.43 \\
\hline$R_{\text {spleen }}$ & 3.31 & 12.5 & 4.65 \\
\hline$R_{\text {intestine }}$ & 7.5 & 13.9 & - \\
\hline$R_{\text {liver }}$ & 10.69 & 16.9 & 11.25 \\
\hline$R_{\text {muscle }}$ & $1.15^{\mathrm{b}}$ & - & - \\
\hline$R_{\text {rest of body }}$ & 11.75 & 7.67 & - \\
\hline$C L_{\text {renal }}(\mathrm{ml} / \mathrm{h})$ & $7.60^{\mathrm{a}}$ & - & - \\
\hline$C L_{\text {int liver }}$ & 685.3 & 15.08 & - \\
\hline$P S_{\text {tumor }}(\mathrm{ml} / \mathrm{h})$ & 5.63 & 14.47 & - \\
\hline$P S_{\text {rest of body }}$ & 106 & 18.67 & - \\
\hline
\end{tabular}

${ }^{\text {a}}$ Fixed values, determined from experimental data.

${ }^{\mathrm{b}}$ Not estimated.

'Values estimated from non-compartmental analysis.

unchanged in the urine. The unchanged SM-406 recovered from feces was negligible.

The tumor concentration-time data were described with a diffusion/permeability-limited model (Figure 3). The model divided the organ into a vascular and extravascular space and captured the data very well. The model yielded an estimate of $5.63 \mathrm{ml} / \mathrm{h}$ for the permeability surface area product. The rest of the body compartment for lumped nonsampled tissues was also characterized with the diffusion-limited model based upon the hypothesis that the heterogeneity of the tissues comprising the compartment was responsible for the polyexponential behavior.

\section{PBPK model validation}

The established PBPK model from $100 \mathrm{mg} / \mathrm{kg}$ dose was used to predict the plasma and tumor concentration-time profile after administration of $30 \mathrm{mg} / \mathrm{kg}$ SM-406 to tumor bearing mice, and the simulated profiles were compared with the observed data (Figure 4). Overall, the model predicted concentration-time profile agreed well with the observed data.

\section{PBPK-PD model}

Smac (second mitochondria-derived activator of caspases) has been identified as an endogenous

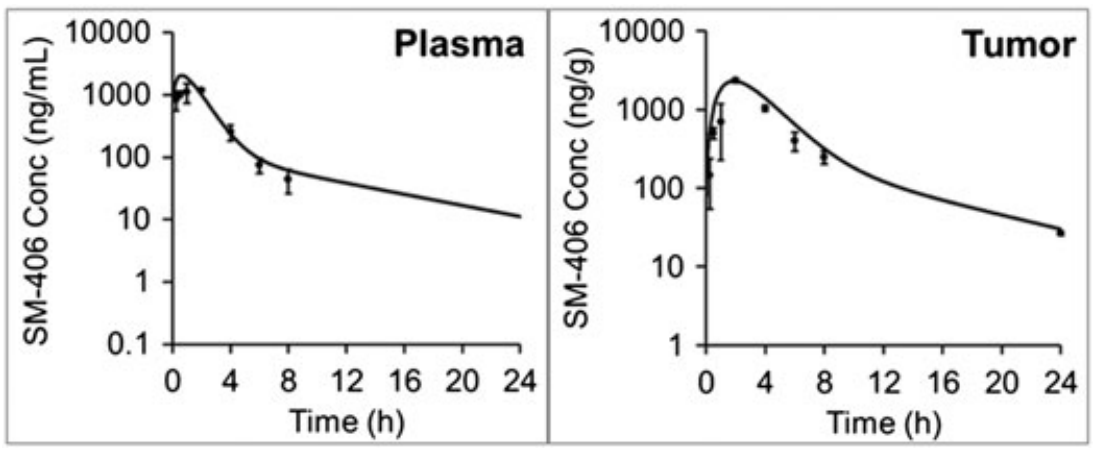

Figure 4. Plasma and tumor concentration-time profiles of SM-406 following an oral dose ( $30 \mathrm{mg} / \mathrm{kg})$ in tumor-bearing mice. Solid circles represent observed concentrations (mean $\pm \mathrm{SD}, n=3)$ and the lines are PBPK model-simulated profiles 
antagonist of XIAP and cIAPs [8,9]. Smac binds to cIAPs to induce their degradation, whose absence activates caspase- 8 , caspase 3 , promotes PARP cleavage and induces cell apoptosis [23,24]. To characterize the effect of SM-406 on cIAP1 inhibition and tumor apoptosis, we built a PBPK-PD model based on the molecular mechanism of apoptosis induction signaling pathways. The schematic illustration of the model is shown in Figure 2. The PBPK estimated tumor SM-406 was used directly to modulate cIAP1 degradation, which further regulated pro-caspase 8 , cleaved PARP (CL-PARP).

The fitted results of onco-protein level using indirect response model are shown in Figure 5. The base levels of cIAP1, pro-caspase 8 and CL-PARP were set at 1 . The simple Emax model was applied to describe the stimulation of cIAP1 degradation by SM-406 concentration in tumor. The effect of cIAP1 on pro-caspase 8 was characterized with a linear model. The accumulation of CL-PARP profile had a negative correlation with the pro-caspase profile so a linear relationship was employed. The model fitted cIAP1 and pro-caspase 8 peak levels showed up between the $2 \mathrm{~h}$ and $6 \mathrm{~h}$ time point. The model caught the trend of the protein level change and described the sparse data reasonably well (Figure 5). The pharmacodynamic parameters generated from the model were listed in Table 3. The synthesis rate constants for the proteins were expressed as normalized amount over time.

In vivo tumor growth in xenograft models is known to be exponential at least in the early phases of tumor development, followed by a linear growth
Table 3. Pharmacodynamic parameter estimates for onco-protein and tumor model

\begin{tabular}{lcll}
\hline Parameter & Unit & Estimated value & $\mathrm{CV} \%$ \\
\hline Onco-protein model & & & \\
$R_{\text {syn_cIAP }}$ & norm.conc. $/ \mathrm{h}$ & 0.45 & 71.4 \\
$E_{\text {max_cIAP }}$ & - & 3.1 & 38.9 \\
$E C_{50 \_c I A P}$ & $\mu \mathrm{M}$ & 12.0 & 48.1 \\
$R_{\text {syn_pCAS8 }}$ & norm.conc. $/ \mathrm{h}$ & 46.2 & 85.1 \\
$K_{\text {pCAS8 }}$ & - & 2.68 & 30.7 \\
Slope_C__PARP & - & 35.9 & 20.0 \\
Tumor growth inhibition model & & \\
$R_{\text {tu_growth }}$ & $1 / \mathrm{h}$ & 0.0068 & 1.00 \\
$K_{\text {tu_apop }}$ & $1 / \mathrm{h}$ & 0.00067 & 1.80 \\
Tu & $\mathrm{mm}{ }^{3}$ & 1260 & 0.60 \\
\hline
\end{tabular}

and a final plateau, as described by a Gompertz model [26-28]. Based on our data, we assumed a maximum tumor volume (TVmax), at which tumor could not grow any more. When the tumor size approached $T V_{\max }$ tumor size increased very slowly [25]. The tumor growth profiles of control, $30 \mathrm{mg} / \mathrm{kg}$ and $100 \mathrm{mg} / \mathrm{kg}$ group were fitted simultaneously with the above mentioned indirect response model using CL-PARP level as a mediator. Both observed data and model predictions are shown in Figure 6 . The estimated parameters were listed in Table 4 . The model adequately represented the observed data and the parameters were estimated with good precision. The control tumor grew approximately linearly before reaching a plateau. The 2-week treatment with $30 \mathrm{mg} / \mathrm{kg}$ or $100 \mathrm{mg} / \mathrm{kg}$ SM-406 effectively delayed the tumor growth, and the delay was well described by the model. After that, the tumor grew in a similar manner to that of

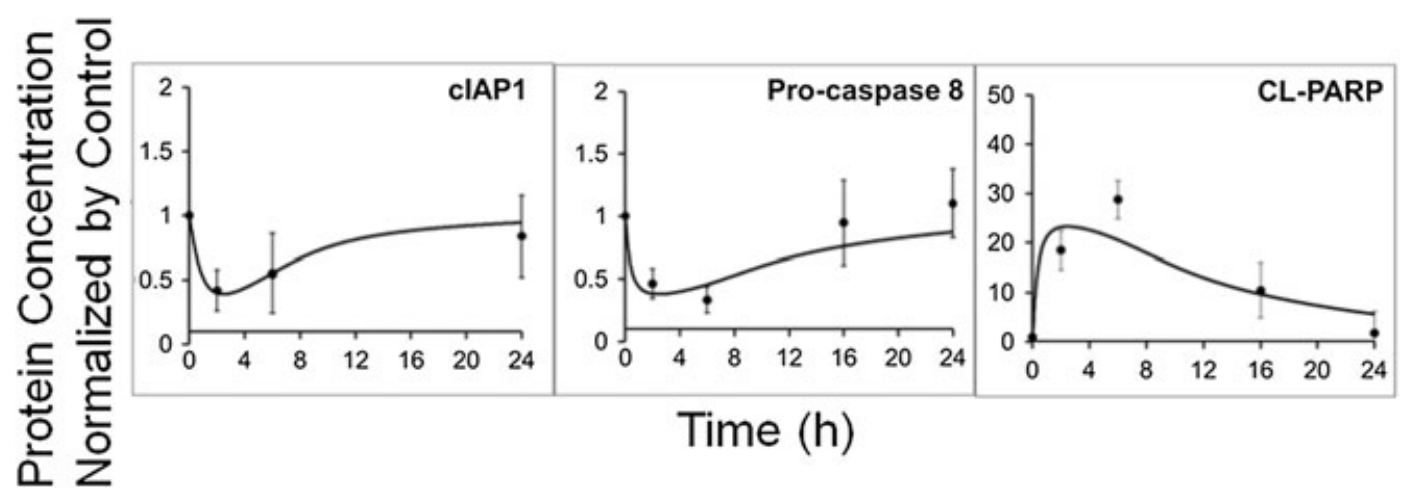

Figure 5. Observed and model-fitted onco-protein levels in tumor-bearing mice following an oral dose of $100 \mathrm{mg} / \mathrm{kg} \mathrm{SM-406.} \mathrm{Solid}$ circles represent measurements and the lines are model-fitted profiles 


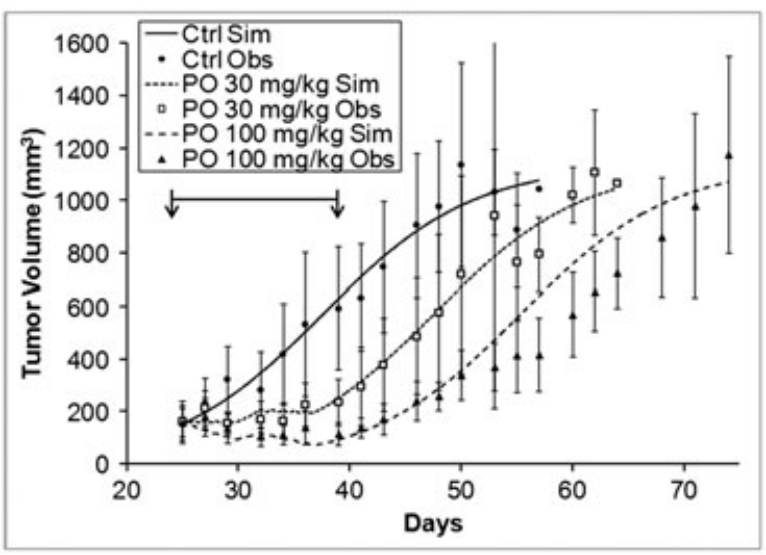

Figure 6. Observed and model-fitted tumor growth profiles in control and treated mice bearing tumors. The mice were dosed daily (5 days/week) with $30 \mathrm{mg} / \mathrm{kg} /$ day or $100 \mathrm{mg} / \mathrm{kg} /$ day SM-406 for 2 weeks. Symbols represent measurements (mean \pm $\mathrm{SD}, n=3$ ) and the lines are model-fitted profiles. Arrows indicate the treatment period

the control as shown in the figure. The model estimated the tumor growth and apoptosis rate constant of $6.8 \times 10^{-3}$ and $6.7 \times 10^{-4} \mathrm{~h}^{-1}$, respectively. The estimated maximum tumor volume was $1260 \mathrm{~mm}^{3}$, which was a reasonable estimate for mice xenografts because of the nutrient limitation. Besides, mice with a tumor over $1000 \mathrm{~mm}^{3}$ need to be euthanized according to the guidelines of the University of Michigan Committee for Use and Care of Animals.

\section{Discussion}

Apoptosis is a critical process in cancer development and progression [4]. One of the most promising targeted therapeutic strategies for cancer therapy is to inhibit the uncontrolled proliferation and induce apoptosis in cancer cells [4]. Apoptosis can be activated through intrinsic and extrinsic pathways, both ending with the activation of caspases to generate the morphology typical of apoptosis [29]. The IAPs negatively regulate the activity of mature caspases and Smac is the endogenous antagonist of IAPs [29]. It has been shown that Smac expression is reduced in tumor progression in many cancer types and probably contributes to intrinsic resistance to chemo- or radiotherapy [29]. SM-406, a potent small molecule mimetic of
Smac synthesized recently, is currently being evaluated in phase I clinical trial for human cancer treatment [14].

The present study characterized the concentrationtime profiles for SM-406 in blood, tumor and typical major organs after oral administration to mice bearing human xenografts, and established a PBPK-PD model to correlate SM-406 tumor concentration with Smac mediated intrinsic apoptosis pathway and tumor growth inhibition.

The development of PBPK models for pharmacokinetic characterization offers many advantages. It helps to gain insight of the pharmacokinetic behavior of the compound in different organs. The results of the present study showed that SM-406 distributed extensively to various organs. The compound was quickly absorbed and distributed after oral administration. In lung, blood, kidney and liver, it reached $C_{\max }$ at $15 \mathrm{~min}$. For all the other tested organs, the $T_{\max }$ was no more than $1 \mathrm{~h}$. Because the concentration-time profiles in most organs appeared to be in parallel with the blood profile, the well stirred model was able to describe the data well (Figure 3). For tumor, both perfusion- and diffusion-limited model were evaluated, and the diffusion-limited model can better depict the concentration-time data. This seems reasonable because the human xenograft tumor was inoculated subcutaneously, the distribution of the compound into tumor may be hindered by different barriers. Some deviation occurred in the prediction of spleen concentration during the elimination phase (Figure 3). We assumed that the drug reached equilibrium instantaneously throughout the entire organ from the incoming plasma flow, so the predicted profile in spleen showed a similar trend compared with plasma. However, the observed data appeared to show that SM-406 was cleared much faster from spleen than from plasma. One explanation for this phenomenon is the unknown clearance mechanism in the spleen, which seems unlikely to our knowledge. Another reason is that spleen was the smallest organ tested in the study, which only had an average weight of $34 \mathrm{mg}$. During sample processing we could only weigh a smaller amount and add 10 folds solution $(\mathrm{v} / \mathrm{w})$ for homogenization, which might introduce a larger error in concentration determination.

The absorption rate constant estimated by PBPK model was $0.9741 / \mathrm{h}$, which is very close to the 
value generated by compartmental analysis. The tissue-to-blood partition coefficients $(R)$ estimated by PBPK were within 1.5-fold difference compared with the AUC ratio from a noncompartmental analysis, indicating a good fit. In the PBPK model, both renal clearance and metabolic biotransformation in the liver were included as the elimination routes. Renal clearance contributed slightly to the total clearance since only about $15 \%$ of unchanged parent compound was recovered compared with the total amount absorbed. The liver intrinsic clearance estimated by PBPK model was $685 \mathrm{ml} / \mathrm{h}$, corresponding to a system clearance of $40.8 \mathrm{ml} / \mathrm{h}$. The system clearance estimated from compartmental analysis was $53.9 \mathrm{ml} / \mathrm{h}$, and the calculated renal clearance was $7.7 \mathrm{ml} / \mathrm{h}$ based on the unchanged compound secreted in urine. The clearance values from PBPK model and compartmental analysis were quite consistent. Compared with the hepatic blood flow of $102 \mathrm{ml} / \mathrm{h}$ in mice [19], SM-406 showed a moderate clearance with an average half life of around $3 \mathrm{~h}$. The developed PBPK model was able to simulate the plasma and tumor concentration-time profiles in the xenograft mice orally administered with a lower dose of $30 \mathrm{mg} / \mathrm{kg}$ (Figure 4). A slight overestimation was observed, suggesting possible saturation of the elimination process since $100 \mathrm{mg} / \mathrm{kg}$ was quite a high dose.

The molecular mechanisms of the intrinsic apoptosis pathway have been studied extensively [29], and the selective targeting of IAPs by SM-406 has been demonstrated at the sub-cellular and molecular level [14]. As a Smac mimetic, SM-406 directly bound to IAPs to prevent their interaction with caspases and resulted in IAPs degradation [14]. Thus, caspases 8, 3 and 9 were activated accordingly, promoting PARP cleavage and inducing apoptosis [14]. To investigate the pharmacokinetic and biomarker relationship, a combined PBPK-PD model was established by linking the tumor concentration-time profiles with oncoprotein levels. This concept was previously introduced in the model of an Hsp90 inhibitor 17-AAG in xenograft mice, and the model provided insightful conclusions on the kinetic and dynamic characteristics of the compound [30]. However, due to the extensive data and analysis required, this kind of model was seldom applied in other compounds. In our model, we not only included the oncoprotein levels, but also connected it with the final efficacy of tumor growth inhibition. In the indirect response model, the synthesis of the onco-proteins was assumed to be zero-order and degradation was by first order. The model adequately described the cIAP1, pro-caspase 8 and CL-PARP protein levels after the single dose administration of SM406 (Figure 5). Upon activation, PARP were cleaved and the cleaved PARP accumulated while cIAP1 level decreased. Although no other caspases data were available, it was very likely after activation they would show a similar trend to CL-PARP based on the knowledge of the underlying mechanisms $[23,24]$. The in vivo tumor growth has been known to follow three stages, exponential growth, linear growth and plateau, which can be described with a Gompertz model [27]. A mathematical model describing this growth behavior has been proposed and tested extensively on a few compounds [28]. In the present study, the tumor growth was monitored after reaching $100 \mathrm{~mm}^{3}$, so a simplified growth model containing a logistic function was employed [25]. The model described the tumor growth in both control and treatment group reasonably well and estimated the growth and apoptosis rate constants of $6.8 \times 10^{-3}$ and $6.7 \times 10^{-4} \mathrm{~h}^{-1}$, which were close to the estimates previously reported [31,32].

In conclusion, an integrated PBPK-PD model was developed to describe the plasma and tissue distribution, onco-protein change and tumor growth inhibition of a small molecule mimetic SM-406 after oral administration in a human xenograft mice model. The PBPK-PD model well described the experimental pharmacokinetic data, the pharmacodynamics biomarker responses and tumor growth. This model may be helpful to predict tumor and plasma SM-406 concentrations in the clinic.

\section{Conflict of Interest}

No conflict of interest was declared.

\section{References}

1. Nicholson DW. From bench to clinic with apoptosis-based therapeutic agents. Nature 2000; 407: 810-816.

2. Ponder BA. Cancer genetics. Nature 2001; 411: 336-341.

3. Lowe SW, Lin AW. Apoptosis in cancer. Carcinogenesis 2000; 21: 485-495.

Biopharm. Drug Dispos. 34: 348-359 (2013)

DOI: $10.1002 /$ bdd 
4. Hanahan D, Weinberg RA. The hallmarks of cancer. Cell 2000; 100: 57-70.

5. Salvesen GS, Duckett CS. IAP proteins: blocking the road to death's door. Nat Rev Mol Cell Biol 2002; 3: 401-410.

6. Deveraux QL, Reed JC. IAP family proteins suppressors of apoptosis. Genes Dev 1999; 13: 239-252.

7. Holcik M, Gibson H, Korneluk RG. XIAP: apoptotic brake and promising therapeutic target. Apoptosis 2001; 6: 253-261.

8. Du C, Fang M, Li Y, Li L, Wang X. Smac, a mitochondrial protein that promotes cytochrome c-dependent caspase activation by eliminating IAP inhibition. Cell 2000; 102: 33-42.

9. Verhagen AM, Ekert PG, Pakusch M, et al. Identification of DIABLO, a mammalian protein that promotes apoptosis by binding to and antagonizing IAP proteins. Cell 2000; 102: 43-53.

10. Srinivasula SM, Hegde R, Saleh A, et al. A conserved XIAP-interaction motif in caspase-9 and Smac/DIABLO regulates caspase activity and apoptosis. Nature 2001; 410: 112-116.

11. Shiozaki EN, Chai J, Rigotti DJ, et al. Mechanism of XIAP-mediated inhibition of caspase-9. Mol Cell 2003; 11: 519-527.

12. Chai J, Du C, Wu JW, Kyin S, Wang X, Shi Y. Structural and biochemical basis of apoptotic activation by Smac/DIABLO. Nature 2000; 406: 855-862.

13. Liu Z, Sun C, Olejniczak ET, et al. Structural basis for binding of Smac/DIABLO to the XIAP BIR3 domain. Nature 2000; 408: 1004-1008.

14. Cai Q, Sun H, Peng Y, et al. A potent and orally active antagonist (SM-406/AT-406) of multiple inhibitor of apoptosis proteins (IAPs) in clinical development for cancer treatment. I Med Chem 2011; 54: 2714-2726.

15. Nestorov I. Whole-body physiologically based pharmacokinetic models. Expert Opin Drug Metab Toxicol 2007; 3: 235-249.

16. Derendorf H, Lesko LJ, Chaikin P, et al. Pharmacokinetic/pharmacodynamic modeling in drug research and development. J Clin Pharmacol 2000; 40: 1399-1418.

17. Zhang T, Hamza A, Cao X, et al. A novel Hsp90 inhibitor to disrupt Hsp90/Cdc37 complex against pancreatic cancer cells. Mol Cancer Ther 2008; 7: 162-170.

18. Laufer R, Paz OG, Di Marco A, et al. Quantitative prediction of human clearance guiding the development of Raltegravir (MK-0518, isentress) and related HIV integrase inhibitors. Drug Metab Dispos 2009; 37: 873-883.

19. Brown RP, Delp MD, Lindstedt SL, Rhomberg LR, Beliles RP. Physiological parameter values for physiologically based pharmacokinetic models. Toxicol Ind Health 1997; 13: 407-484.
20. Baxter LT, Zhu H, Mackensen DG, Jain RK. Physiologically based pharmacokinetic model for specific and nonspecific monoclonal antibodies and fragments in normal tissues and human tumor xenografts in nude mice. Cancer Res 1994; 54: 1517-1528.

21. Wang P, Ba ZF, Burkhardt J, Chaudry IH. Traumahemorrhage and resuscitation in the mouse: effects on cardiac output and organ blood flow. Am J Physiol 1993; 264: H1166-H1173.

22. D'Argenio DZ, Schumitzky A, Wang X. ADAPT 5 User's Guide: Pharmacokinetic/Pharmacodynamic Systems Analysis Software. Biomedical Simulations Resource: Los Angeles, 2009.

23. Dai Y, Liu M, Tang W, et al. A Smac-mimetic sensitizes prostate cancer cells to TRAIL-induced apoptosis via modulating both IAPs and NF-kappaB. BMC Cancer 2009; 9: 392.

24. Roth W, Reed JC. Apoptosis and cancer: when BAX is TRAILing away. Nat Med 2002; 8: 216-218.

25. Cao X. Simultaneously targeting hypoxic cancer cells by hsp90 inhibitor and glycolysis inhibitor in pancreatic cancer therapy. In College of Pharmacy. The Ohio State University: Columbus, 2007; 239.

26. Bissery MC, Vrignaud P, Lavelle F, Chabot GG. Experimental antitumor activity and pharmacokinetics of the camptothecin analog irinotecan (CPT11) in mice. Anticancer Drugs 1996; 7: 437-460.

27. Norton L, Simon R. Growth curve of an experimental solid tumor following radiotherapy. J Natl Cancer Inst 1977; 58: 1735-1741.

28. Simeoni M, Magni P, Cammia C, et al. Predictive pharmacokinetic-pharmacodynamic modeling of tumor growth kinetics in xenograft models after administration of anticancer agents. Cancer Res 2004; 64: 1094-1101.

29. Martinez-Ruiz G, Maldonado V, Ceballos-Cancino G, Grajeda JP, Melendez-Zajgla J. Role of Smac/ DIABLO in cancer progression. J Exp Clin Cancer Res 2008; 27: 48.

30. Xu L, Eiseman JL, Egorin MJ, D'Argenio DZ. Physiologically-based pharmacokinetics and molecular pharmacodynamics of 17-(allylamino)-17-demethoxygeldanamycin and its active metabolite in tumorbearing mice. J Pharmacokinet Pharmacodyn 2003; 30: 185-219.

31. Yamazaki S, Nguyen L, Vekich S, et al. Pharmacokinetic-pharmacodynamic modeling of biomarker response and tumor growth inhibition to an orally available heat shock protein 90 inhibitor in a human tumor xenograft mouse model. J Pharmacol Exp Ther 2011; 338: 964-973.

32. Salphati L, Wong H, Belvin M, et al. Pharmacokinetic-pharmacodynamic modeling of tumor growth inhibition and biomarker modulation by the novel phosphatidylinositol 3-kinase inhibitor GDC-0941. Drug Metab Dispos 2010; 38: 1436-1442. 